\title{
Die Gründung der Hebammenschulen in der Schweiz im 18. Jahrhundert
}

(mit besonderer Berücksichtigung der bernischen Verhältnisse)

\author{
Von Hans Jenzer, Bern
}

Die Gründungsgeschichte der Hebammenschulen in der Schweiz ist noch nicht im Zusammenhang geschildert worden, wohl aber gibt es einige gute Einzeldarstellungen. So berichtet KerN ${ }^{1}$ über die Verhältnisse in Basel, auf die auch Staehelin ${ }^{2}$ kurz eingeht, und FlüEler ${ }^{3}$ beschreibt das Hebammenwesen im alten Stand Solothurn. Der Hebammenschule von Yverdon wird in ausführlicher, wenn auch nicht ganz vollständiger Art von OLIVIER $^{4}$ gedacht. Die Geschichte des Medizinalwesens in Zürich, das im 18. Jahrhundert keine eigentliche Hebammenschule besaß, wird von WeHrLI $^{5}$ und MeIER-Ahrens ${ }^{6}$ dargestellt; aufschlußreich ist auch die Rede Rahns ${ }^{7}$, die er 1784 anläßlich der Eröffnung des Zürcherischen Seminariums hielt. MicHEL ${ }^{8}$ schildert die Ereignisse in Luzern, worüber uns auch HorN ${ }^{9}$ in der Einleitung seines Buches über die Hebammenkunst berichtet. Die geschichtliche Entwicklung des Medizinalwesens in Genf schließlich beschreibt GaUtIER ${ }^{10}$. In verschiedenen Publikationen werden

1 Hans Kern, Zur Geschichte des Hebammenwesens in Basel, Basel 1929.

2 Andreas Staehelin, Geschichte der Universität Basel 1632-1818, Basel 1957.

3 Benno Flüeler, Ärzte, Apotheker, Chirurgen und Hebammen im alten Stande Solothurn, Solothurn 1951.

4 Eugène Olivier, Médecine et santé dans le Pays de Vaud au XVIII ${ }^{e}$ siècle 1675-1798, 1.Band, Lausanne 1962.

5 Gustav Adolf Wehrli, Die Krankenanstalten und die öffentlich angestellten Ärzte und Wundärzte im alten Zürich, Mitteilungen der Antiquarischen Gesellschaft in Zürich XXXI (1934) Heft 3.

${ }^{6}$ MeIer-Ahrens, Geschichte des Zürcherischen Medizinalwesens, 1.Teil Zürich 1838, und 2.Teil Basel 1840.

7 Johann Conrad Rahn, Die innere Einrichtung des zur Bildung geschickter Landärzten und tüchtigen Landhebammen, wie auch zu einer Privatverpflegung armer Kranker in Zürich errichteten Seminariums, Gazette de Santé 1784, 332.

8 Theodor Michel, Bader, Scherer, Chirurgen, Hebammen und Apotheker im alten Luzern (1300-1798), Geschichtsfreund 132 (Stans 1931) 207.

9 Johann von HoRn, Hebammenkunst durch Fragen und Antworten vorgetragen, 6. Auflage, Luzern 1776.

10 LÉon Gautier, La Médecine à Genève jusqu'à la fin du 18ième siècle, Genève 1906. 
die bernischen Verhältnisse geschildert, so von ThurnheER ${ }^{11}$, JAUssi ${ }^{12}$, Hintzsche ${ }^{13}{ }^{14}$, Rennefahrt und Hintzsche ${ }^{15}$ und Jenzer ${ }^{16}$, doch wird nirgends eingehend auf die Gründungsgeschichte der Hebammenschule in Bern eingegangen; dieses nachzuholen bildet das Hauptanliegen der vorliegenden Arbeit.

Die Geburtshilfe lag im 17. Jahrhundert fast ausschließlich in den Händen der Hebammen. Eigentliche Hebammenschulen gab es aber mit einer Ausnahme nicht, sondern die Frauen, die sich dem Beistand der Gebärenden widmen wollten, erlernten ihr Handwerk meist bei einer älteren Kollegin. Vielerorts wurden die Hebammen durch die Stadtärzte unterrichtet. 1630 ist das Gründungsjahr der ersten Gebärabteilung im Hôtel-Dieu in Paris, wo die angehenden Hebammen Lehrkurse bei einer maîtresse de sagefemme erhielten. Im Laufe des 17. und 18. Jahrhunderts ging die Geburtshilfe namentlich infolge der glänzenden Leistungen der französischen Chirurgen allmählich an das männliche Geschlecht über, und die Hebammen wurden in die zweite Position gedrängt. Aus dem Stand der französischen Chirurgen entwickelte sich der «accoucheur», und Frankreich sollte auf dem Gebiet der Geburtshilfe noch bis weit ins 19. Jahrhundert hinein führend sein. Doch auch als die Ärzte die Führung der Geburtshilfe übernommen hatten, blieb sie noch lange in ihrem Grundcharakter eine Hebammenkunst und fand den Anschluß an die Medizin erst in der zweiten Hälfte des 19.Jahrhunderts. 1728 (also fast 100 Jahre nach Paris) erfolgte in Straßburg die Gründung einer Gebäranstalt für den Hebammenunterricht, deren erster Leiter Johann JAGob Fried wurde. 1737 erweiterte man sie zu einem klinischen Institut für Studierende der Medizin und für Hebammenschülerinnen. In der Folge wurde die Straßburger Hebammenschule die Musterschule, nach deren Vorbild man in Deutschland und in den umliegenden Ländern gleiche Institute gründete, so 1751 in Göttingen und Berlin, 1754 in Moskau, 1755 in Stockholm, 1761 in Kopenhagen, um nur

11 Yvonne Thurnheer, Die Stadtärzte und ihr Amt im alten Bern, Bern 1944.

12 Ruth JAussi, Das Medizinische Institut in Bern (1797-1805), Bern 1944.

13 Erich Hintzsche, Die geschichtliche Entwicklung anatomischer Arbeit in Bern bis zur Gründung der Hochschule, Bern 1942.

14 ERICH HiNtzsche, Über medizinischen Unterricht im alten Bern, Bern 1951.

15 Hermann Rennefahrt und Erich Hintzsche, 600 Jahre Inselspital Bern (1354-1954), Bern 1954.

16 Hans Jenzer, Johann Friedrich v. Herrenschwand, ein Berner Arzt im 18. Jahrhundert, im Druck. 
einige zu nennen (Literatur u. a. bei Fasbender ${ }^{17}$, PuschmanN ${ }^{18}$, Hirsch $^{19}$, Wieger ${ }^{20}$, Osiander ${ }^{21}$ ). Im ausgehenden 18. Jahrhundert entstanden zudem zahlreiche Lehrbücher über die Geburtshilfe und die Hebammenkunst: Steidele, Josephi, Levret, Zandt, Aepli, Venel, von Horn, Hirzel u. a.

Das war die Situation, als auch in der Schweiz an die Gründung von Hebammenschulen gedacht wurde, und es ist nicht vérwunderlich, daß die erste schweizerische Hebammenschule in Basel, also in nächster Nachbarschaft von Straßburg, entstehen sollte. Da Kern ${ }^{1}$ und Staehelin ${ }^{2}$ ihre Entstehung eingehend beschrieben haben, beschränke ich mich auf einen kurzen Hinweis. Schon 1766 trat der Arzt Johans Rudolf Hess für eine Revision und Neuordnung des Hebammenwesens ein, und 1769 wurde ein Projekt für eine Hebammenschule mit einem Hebammenmeister eingereicht. Gleichzeitig hieß die Obrigkeit eine neue Hebammenordnung nach dem Muster Straßburgs gut. Die Hebammenschule in Basel wurde 1771 eröffnet; Johann Rudolf $\mathrm{Heß}$ war der erste Hebammenmeister. Da schon 1773 alle Schülerinnen die Schule nach bestandenem Examen verlassen hatten (das Einzugsgebiet beschränkte sich auf die Stadt Basel und die umliegenden Gemeinden) und keine neuen Lehrtöchter vorhanden waren, mußte die Schule vorübergehend geschlossen werden, und nur nach Bedarf wurden neue Kurse angesetzt; dagegen wurden im Theatrum anatomicum und im Spital die Demonstrationen zur Fortbildung der amtierenden Hebammen weitergeführt.

Von Flüeler ${ }^{3}$ werden wir über die Verhältnisse in Solothurn orientiert. Dort wurde 1779 eine Hebammenschule gegründet; als Hebammenmeister konnte Johann JACOB WIRz gewonnen werden, der sich bald weit herum einen Namen als tüchtiger Geburtshelfer machte. Dem Unterricht diente das Helfemutterbüchlein des Johann von Horn ${ }^{9}$. Leider erfahren wir nicht, wie lange diese Schule existierte und wie viele Schülerinnen sie ausgebildet hat. Es müssen aber vorwiegend nur die Hebammen der Stadt und der

17 Heinrich Fasbender, Geschichte der Geburtshilfe, Jena 1906 (Nachdruck Hildesheim 1964).

18 Theodor Puschmann, Geschichte des medizinischen Unterrichts, Leipzig 1889.

19 August Hirsch, Geschichte der medicinischen Wissenschaften in Deutschland, München/ Leipzig 1893.

20 Friedrich Wieger, Die Geschichte der Medizin und ihrer Lehranstalten in Straßburg (1497-1872), Straßburg 1885.

21 Friedrich Benjamin Osiander, Denkwürdigkeiten für die Heilkunde und Geburtshülfe, Göttingen 1794. 
näheren Umgebung gewesen sein, und damit herrschten wohl in Solothurn ähnliche Verhältnisse wie in Basel. Die Geburtshilfe auf dem Lande blieb bis Ende des 18. Jahrhunderts ein Sorgenkind der Regierung von Solothurn. Zwar hatte die Obrigkeit schon 1775 verfügt, daß die einzelnen Gemeinden der Helfmutter (wie die Hebamme im Solothurnischen genannt wurde) ein Wartgeld ausrichten sollten.

Die Hebammenschulen von Yverdon (1778) und Bern (1781) müssen zusammen betrachtet werden. Damals wurde das Gebiet des heutigen Kantons Waadt von Bern regiert, und die Gnädigen Herren von Bern erteilten alle gesundheitspolizeilichen Weisungen. Sosehr die Initiative Venels für die Gründung der Hebammenschule von Yverdon zu würdigen ist, gehen doch die beiden Schulen Bern und Yverdon auf die gleichen Quellen zurück, was von Olivier ${ }^{4}$ nicht eindeutig dargestellt wurde.

Die ersten Hinweise dafür, daß sich die bernische Regierung mit der Gründung einer Hebammenschule befaßt hat, finden wir zu Beginn des Jahres 1765. Am 4. Januar dieses Jahres wurde eine «Landesalmosen-Revisions-Commission» bestellt, die nach den Ursachen der Bevölkerungsabnahme in gewissen Gegenden der bernischen Lande forschen sollte. Schon am 12. Februar 1765 stellte diese Kommission in einem Bericht fest, der Grund der Bevölkerungsabnahme liege im Mangel an tüchtigen Wehemüttern und erfahrenen Landärzten ${ }^{22}$. Am 12. März 1765 befaßte sich der Große Rat eingehend mit diesem Problem ${ }^{23}$. Er beauftragte gleichentags den Sanitätsrat die Frage zu studieren, wie dem Mangel an Ärzten und Hebammen auf dem Lande abgeholfen werden könne; dazu möge er erfahrene Medicos und Chirurgos zu Rate ziehen ${ }^{24}$. Am 20. März 1765 beschloß der Sanitätsrat, Albrecht von Haller den Auftrag zu erteilen, ein Gutachten darüber auszuarbeiten ${ }^{25}$. Die mit dem Datum vom 26. April 1765 versehene Schrift Hallers trägt den Titel: Gedanken über die Verbesserung der zur Kunst zu Heilen einschlagende Wissenschaften ${ }^{26}$. Haller befaßte sich darin in erster Linie mit der Ausbildung von Ärzten, die ich übergehe. Über die Hebammen schreibt er, sie seien nur imstande «kleine Dienste zu leisten und das von der Natur ihnen angebotene Kind zu empfangen, nicht aber

22 Staats-Archiv Bern (StAB) B XI 132, S. 63-68 (Sanitätswesen 18. Jahrhundert Nr. 100).

${ }^{23}$ StAB, Ratsmanual 274, S. 219-221.

${ }^{24}$ StAB, B XI 132, S. 55-58 (Sanitätswesen XVIII Jahrhundert Nr. 100).

25 StAB, Manual des Sanitätsrates Nr. 41 und B XI 132, S. 45-48.

${ }^{26}$ StAB, B XI 132, S. 67-96. 
das Geringste bey unrichtigen Stellungen und eingeklemmten Köpfen anzurahten oder geschikt anzugreifen ». Er weist dann auf die guten Erfolge der Straßburger Hebammenschule hin, der unser Land nichts Gleichwertiges entgegenzustellen habe. Die Ausbildung der Hebammen gehöre in ein größeres Spital, und dazu müßte wie in Straßburg oder Göttingen ein tüchtiger Hebammenmeister bestellt werden, der selbst die Handgriffe kenne und sie auch den lernenden Hebammen zeigen könne. Dieser müßte ferner bei allen Geburten im Spital anwesend sein, die schweren selbst leiten und zugleich die anwesenden Schülerinnen über alle Gefahren und ihre Abwendung unterrichten. In der Stadt Bern sollte keine Hebamme zugelassen werden, die die Schule nicht während zweier Jahre besucht hätte. Und diese Stadthebammen sollten dann selbst Lehrerinnen werden für die Landhebammen, da man nicht alle Landhebammen nach Bern berufen könne. Die Vorschläge Hallers wurden am 13.Juni 1765 vom Sanitätsrat gutgeheißen und an die Obrigkeit weitergeleitet ${ }^{27}$. Schon vorher war durch Tissot in Lausanne ein ähnlicher, aber viel weitergehender Plan eingereicht worden. Er trägt das Datum vom 12. Februar 1765 und ist betitelt: Essai sur les moyens de perfectionner les études de médecine ${ }^{28}$. Wie Tissot in der Einleitung selbst bemerkt, ist er vom Präsidenten des Sanitätsrates dazu aufgefordert worden, dieses Gutachten auszuarbeiten; in den Manualen des Sanitätsrates fehlt allerdings eine entsprechende Notiz. Das sehr umfangreiche, 36 Seiten starke Manuskript deckt sich inhaltlich weitgehend mit den Richtlinien, die Tissot in seinem Buch Avis au peuple sur la santé entwickelt hat. Über die Ausbildung der Hebammen schreibt er, es sei für diese vor allem wichtig, eine normale Geburt richtig beenden zu können und eine abnormal verlaufende frühzeitig zu erkennen: «il faudra leur marquer les bornes où elles doivent s'arrêter, et dans lesquelles il faut recourir aux accoucheurs des villes». Sie sollten die Symptome einer Schwangerschaft kennen und lernen, wie ein Abort zu verhüten sei; auch in der Pflege der Neugeborenen sollten sie sich auskennen. Seine Ausführungen gipfeln im Vorschlag, in Bern eine Medizinschule unter der Führung Hallers zu gründen, an der auch die Hebammen ausgebildet werden könnten. Haller hatte in seinem Bericht den Plan Tissots abgelehnt. Er glaubte, er sei im Moment wegen fehlender finanzieller Mittel nicht durchführbar. Man müsse sich mit dem begnügen, was realisierbar sei, mit Stipendien für

27 StAB, Manual des Sanitätsrates, Band 42, S. 57-59.

28 StAB, B XI 132. 
angehende Ärzte und Wundärzte, mit kleinen Sonderkursen am Inselspital sowie mit einem einjährigen Praktikum am Inselspital für angehende Wundärzte. Am 10. März $1766^{29}$ erhielt der Sanitätsrat von der Obrigkeit den Auftrag, den Vorschlag Hallers, den er gut befunden habe, zu bereinigen und präzise Vorschläge zu unterbreiten. Es wurden zur Bestreitung der jährlichen Unkosten 600 Kronen bewilligt. Darauf arbeitete der Sanitätsrat einen Entwurf aus, betitelt: Project-Reglement zur Bildung guter Ärzten, Chirurgi und Hebammen zu Stadt und Land ${ }^{30}$. Darin wird im Abschnitt 3 über den zu wählenden Hebammenmeister folgendes gesagt: Zum Hebammenmeister sollte nur ein Chirurg gewählt werden, der entweder Burger der Stadt Bern oder Kantonsangehöriger sei («Landeskind», wie der damalige Ausdruck lautete); er müsse die Hebammenkunst schon während mindestens drei Jahren ausgeübt haben und sollte den lernenden Weibspersonen wenigstens jede Woche einmal eine Vorlesung halten über die Geburten und die Besorgung der Kindbetterinnen. Bei allen Geburten im Spital hätte er anwesend zu sein, die schweren müßte er selbst leiten und die Anfängerinnen auf alle auftretenden Gefahren aufmerksam machen und sie in den gebräuchlichen Handgriffen unterrichten. Doch sollte er nur Burger oder Landeskinder unterrichten und jährlich einmal im Theatro anatomico eine Section eines weiblichen Körpers vornehmen und den Schülerinnen «die innerlichen zur Erzeugung und Geburt gehörigen Teile vorzeigen und demonstrieren ». Sein jährliches Gehalt wurde auf 150 Kronen festgesetzt. In einem Nachtrag wurde weiter ausgeführt, daß die Landstädte und Landschaften ehrliche und geschickte Weibspersonen, die sich zu Hebammen ausbilden lassen möchten, in die Hauptstadt (der damals übliche Ausdruck für die Stadt Bern) schicken sollten, wo sie während dreier Jahre unentgeltlich dem Unterricht des Hebammenmeisters folgen könnten. Nach Bedarf sollte der Unterricht auch in französischer Sprache erteilt werden. Die auswärtigen Schülerinnen könnten im großen Spital Gemach, Bett, Speis und Trank umsonst genießen. Dieses Projekt entspricht genau den Vorschlägen Hallers.

Leider verflog der anfängliche Elan rasch. Das Projekt verschwand offenbar in einer Schublade. Erschwerend für die Gründung einer Hebammenschule in Bern war von Anfang an der Umstand, daß das Projekt immer mit einer zu gründenden Ausbildungsstätte für junge Ärzte und Wundärzte ge-

29 StAB, Manual des Sanitätsrates, Band 42, S. 355/56.

${ }^{30}$ StAB, B XI 132 und Manual des Sanitätsrates, Band 43, S. 96-104. 
koppelt war, ja daß die Hebammenschule eigentlich immer nur als Anhängsel dieser «Pflanzschule für junge Ärzte» gedacht war. Es wurden auch Bedenken laut, ob die zu gründende Ärzteschule mit nachfolgender Patentierung von Land- und Wundärzten im Einklang stehe mit den Privilegien der chirurgischen Societät (der Vereinigung der bernischen Wundärzte). Im März 1768 wurde das Projekt nochmals an den Sanitätsrat zur Überprüfung geschickt ${ }^{31}$. Die Berner Regierung scheute sich immer davor, altes Recht zu verletzen. Nachdem aber festgestellt worden war, daß die Statuten der Chirurgischen Societät nichts enthielten, das der Gründung einer Medizinschule in Bern entgegenstünde, arbeitete der Sanitätsrat einen neuen, fast gleichlautenden Entwurf aus, der in den Manualen enthalten ist $^{32}$. Auch das Inselcollegium gab zu dem Projekt seine Zustimmung ${ }^{33}$, glaubte aber, daß die Entlöhnung des Hebammenmeisters zu niedrig angesetzt sei und daß es schwer halten würde, für so wenig Geld einen geeigneten Mann zu finden. Und nun wurde es wieder still um die Hebammenschule bis zum Jahr 1776. Die Anfrage der Obrigkeit, ob die Tätigkeit eines Hebammenmeisters mit dem Amt eines hauptamtlichen Chirurgen des Inselspitals zu vereinen sei, wurde gemeinsam vom Inselcollegium und vom Sanitätsrat abgelehnt (Beschluß vom 26. Dezember $1776^{34}$ ). Und nun wurde von Johann Friedrich von Herrenschwand aus Murten ein neues Gutachten eingeholt, über das anderwärts ausführlich berichtet wird ${ }^{16}$. Diese Schrift, die Anfang Januar 1777 eingereicht wurde ${ }^{35}$, trägt den Titel: Sur les écoles d'accouchement dans l'étranger et la nécessité d'en établir dans les pays qui en manquent. Avec l'ouverture d'un moyen de créer avec des frais modiques dans la ville de Berne une école qui nantiroit à perpétuité les Etats de Leurs Excellences de sages-femmes instruites, d'accoucheurs habiles... $\gg{ }^{36}$ Auch Herrenschwand verband seine Hebammenschule mit einer Ausbildungsstätte für Landärzte. Einleitend beschrieb er die Schulen von Paris, Straßburg und Berlin und erwähnte ihre guten Erfolge. Aber es komme nicht nur darauf an, gute Hebammen auszubilden, sondern gleichzeitig müßte auch für gute Geburtshelfer gesorgt werden. Denn wenn es genügend Geburtshelfer gebe, so sei die Aufgabe der Hebamme einfach und könne auf die Leitung

31 StAB, Manual des Sanitätsrates, Band 44, S. 120/21.

32 StAB, Manual des Sanitätsrates, Band 47, S. 83-103.

33 StAB, B XI 132.

34 StAB, Manual des Sanitätsrates, Band 57, S. 82.

35 StAB, Manual des Sanitätsrates, Band 57, S. 118.

36 StAB, B XI 132. 
normaler und das rechtzeitige Erkennen von schwierigen Geburten beschränkt werden: «L'expérience a prouvé que de vouloir étendre la sphère de la sage-femme c'est lui ouvrir une carrière où elle se perd et fait avec confiance mille maux.» Mit zwei Unterrichtsstunden täglich (je eine Stunde Theorie und eine Stunde praktische Übung im Gebärsaal) könnte eine Hebamme in drei Monaten genügend ausgebildet werden. Der Unterricht der Hebammen wäre mit demjenigen für junge Ärzte und Chirurgen zu kombinieren.

In diese Zeit fällt nun die Eingabe von Venel, der beabsichtigte, in Yverdon eine Hebammenschule zu eröffnen (siehe Olivier ${ }^{4}$ ). Schon 1770 hatte Venel seinen ersten Vorschlag eingereicht: Mémoire sur l'établissement d'une école pour instruire des sages-femmes dans leur art projettée a Yverdon». ${ }^{37}$ Fast gleichzeitig machte Secrétan einen Vorstoß, für das Waadtland in Lausanne eine Hebammenschule zu gründen ${ }^{38}$. Da der Boden in Bern durch die jahrelange Diskussion gut vorbereitet war, ging der Wunsch Venels, der im Dezember 1776 sein zweites Projekt einreichte (in der Zwischenzeit war er als Leibarzt des Fürsten Ротоскі in Polen gewesen), rasch in Erfüllung. In den Darstellungen Oliviers, der auf die Vorgeschichte in Bern nur andeutungsweise eingeht, wird diesem Umstand zu wenig Rechnung getragen. Ohne die Verdienste Venels irgendwie schmälern zu wollen, wäre er doch in Bern mit seinen Plänen nicht so schnell durchgedrungen, wenn die Berner Regierung nicht von der Notwendigkeit der Hebammenschulen überzeugt gewesen wäre. Es darf zudem vermutet werden, daß die Gründung einer Hebammenschule in Yverdon der bernischen Obrigkeit schon deshalb willkommen sein mußte, als damit für die Hebammenschule in Bern die nötigen Erfahrungen gesammelt werden konnten. So konnte der Sanitätsrat am 20. Mai 1777 den Landvögten im welschen Land die Mitteilung machen, daß in Iferten eine Hebammenschule vorgesehen sei ${ }^{39}$. Eine gleiche Mitteilung ging am 28. April 1777 an den Landvogt von Yverdon, und am 31.Dezember 1777 wurde Venel vom Sanitätsrat mitgeteilt, er könne mit dem Unterricht sofort beginnen ${ }^{40}$. Die Hebammenschule in Yverdon wurde am 2.Februar 1778 eröffnet, und am 18. April 1778 konnte Venel das Diplom an 18 Hebammen erteilen, die den Kurs und das nach-

\footnotetext{
$37 \mathrm{ib.}$

38 ib.

39 StAB, Manual des Sanitätsrates, Band 57, S. 176 und 178.

40 StAB, Manual des Sanitätsrates, Band 59, S. 35.
} 
folgende Examen erfolgreich absolviert hatten. Der Sanitätsrat in Bern unterzeichnete die Diplome und ernannte die Hebammen zu «Maîtresse sage-femme ».41 Die Kurse wurden in der Folge fast jeden Winter fortgesetzt, wenn auch mit stark verminderten Schülerinnenzahlen. 1780 verlegte Venel die Hebammenschule nach Orbe, wo er unterdessen seine orthopädische Klinik gegründet hatte. Zahlreiche Vermerke in den Manualen des Sanitätsrates berichten über die weitere erfolgreiche Tätigkeit Venels, und auch der Dank der Obrigkeit blieb nicht aus.

Unterdessen ging die Diskussion in Bern weiter. Im Juni 1778 erhielt der Stadtarzt Dr. Tribolet den Auftrag, das Buch Venels über die Hebammenkunst ins Deutsche zu übersetzen ${ }^{42}$. Und endlich, am 22. März 1781, erstellte der Sanitätsrat ein neues «Gutachten zur Errichtung einer Hebammenschule in hiesiger Hauptstadt $»{ }^{43}$ Er wies darin auf die Erfolge der Hebammenschule in Yverdon hin, wo bis heute zahlreiche tüchtige Wehemütter ausgebildet wurden; dabei koste die Schule der Regierung nicht einmal 500 Franken jährlich. Dieses Mal billigte die Regierung den Vorschlag und beauftragte am 2.April 1781 den Sanitätsrat, die weiteren Schritte zu unternehmen ${ }^{44}$, nachdem am 5. April Venel mit seinen Schülerinnen nach Bern gekommen war, um sie vom Inselcollegium und vor dem Sanitätsrat prüfen zu lassen, und allseitigen Beifall gefunden hatte. Vor allem mußte mit der Inseldirektion verhandelt werden, da diese noch 1777 behauptet hatte, in der Insel wäre weder Platz für die Hebammenschule noch für die Schülerinnen. Die Unterredung führte zu einer Einigung. Im unbenützten Zimmer für Waisenkinder wurden 12 Betten für die 24 Schülerinnen bereitgestellt, und auch über das Kostgeld konnte man sich einigen ${ }^{45}$. Als Hebammenmeister wurde der Operator KöNIG gewählt (4.Juli 1781) ${ }^{46}$, und zugleich erfolgte eine Mitteilung an alle Städte und Dorfschaften des «teutschen Landes», daß in Bern am 1.Dezember 1781 eine Hebammenschule eröffnet werde. Am 26. Februar 1782 konnten 23 Schülerinnen nach erfolgreich bestandenem Examen diplomiert werden ${ }^{47}$. Am 27.Februar 1782 erfolgte ferner die offizielle Mitteilung an die Stände

41 StAB, Manual des Sanitätsrates, Band 59, S. 111-115.

42 StAB, Manual des Sanitätsrates, Band 59, S. 166.

43 StAB, Manual des Sanitätsrates, Band 63, S. 192-195.

44 StAB, Manual des Sanitätsrates, Band 63, S. 217-220.

45 StAB, Manual des Sanitätsrates, Band 63, S. 252-256.

46 StAB, Manual des Sanitätsrates, Band 64, S. 4-10.

47 StAB, Manual des Sanitätsrates, Band 64, S. 269-274. 
Zürich, Luzern, Uri, Schaffhausen, Basel und Solothurn, ferner an Pünten, Mühlhausen, Wallis, Genf und St.Gallen, daß in Bern eine Hebammenschule bestehe; gleichzeitig wurde ein Exemplar des durch Dr. Tribolet übersetzten Hebammenlehrbuches von Venel überreicht ${ }^{48}$. Die Schule nahm einen guten Fortgang. 1783 konnte an weitere 18 Hebammen das Diplom erteilt werden, und 1784 waren es immer noch 13. Nach drei Jahren stellte der Sanitätsrat zu Handen der Obrigkeit fest, daß bis jetzt 54 tüchtige Wehemütter ausgebildet worden seien und daß das Werk so lange fortgesetzt werden sollte, bis in jedem Kirchenspiel mindestens 1 bis 2 Hebammen vorhanden wären ${ }^{49}$. Es muß hier noch festgestellt werden, daß die Organisation der bernischen Hebammenschule genau den Vorschlägen Herrenschwands folgte. Vom ursprünglich gutgeheißenen Plan Hallers sprach niemand mehr. Dieser hatte vorwiegend die Hebammen der Stadt Bern ausbilden wollen mit einer Kursdauer von zwei Jahren; Landhebammen hätten in einem dreijährigen Kurs nur in beschränktem Umfange zugelassen werden können. Herrenschwand dagegen beschränkte sich auf einen Kurs von drei Monaten, sah aber gleichzeitig vor, seine Schule allen Hebammenschülerinnen zu Stadt und Land offenzuhalten, um so, und das war sein wesentliches Anliegen, vor allem die medizinische Versorgung der Landbevölkerung zu verbessern.

An den übrigen Orten der Schweiz bestanden im 18. Jahrhundert keine eigentlichen Hebammenschulen. In der Stadt Zürich oblag dem Stadtarzt der Unterricht und die Prüfung der Hebammen (siehe Wehrli ${ }^{5}$ und MeierAhrens $\left.{ }^{6}\right)$. Erst mit der Gründung des Medizinisch-chirurgischen Instituts 1782 und des Seminars 1784 (siehe Rahn ${ }^{7}$ ) wurde auch vereinzelten Landhebammen ermöglicht, in der Stadt dem Unterricht des Stadtarztes zu folgen. Lange noch aber sollte die Ausbildung der Landhebammen eine große Sorge der Regierung bleiben.

Auch in Luzern kam es nicht zur Gründung einer Hebammenschule im Ausmaß von Bern und Yverdon. Hier wirkte seit 1768 die weit über die Stadtgrenze hinaus berühmte Maria Victoria Hartenbein als Stadthebamme und bildete sicher manche gute Hebamme aus. Michel ${ }^{8}$ spricht zwar von einer Hebammenschule, doch fehlen genaue Angaben darüber, und auch v.Horn schreibt in der Vorrede seines Buches ${ }^{9}$, daß in Luzern eine Hebammenschule errichtet wurde und daß die nach Luzern berufene, bei

48 StAB, Manual des Sanitätsrates, Band 64, S. 275.

49 StAB, Manual des Sanitätsrates, Band 68, S. 268-271. 
Fried in Straßburg ausgebildete Frau Hartenbein als öffentliche Lehrerin bestellt sei. Sonst vernehmen wir aber nichts darüber, und wir können nur vermuten, daß es sich mehr um eine rein städtische Einrichtung gehandelt habe, die sich auf die Hebammen der Stadt Luzern beschränkte.

In Genf gab es nach Gautier ${ }^{10}$ seit 1746 Demonstrationskurse für Hebammen, die bis zum Ende der Republik beibehalten wurden. Von einer eigentlichen Hebammenschule ist aber nicht die Rede.

Zusammenfassend stellen wir fest, daß die Hebammenschulen in Basel (1771) und in Solothurn (1779) sich kaum über den Stadtbereich hinaus erstreckten und ihre Tätigkeit nach einiger Zeit wegen Schülerinnenmangels wieder einstellen mußten. Der Schule Venels in Yverdon (1778) war ein größerer Erfolg beschieden, weil sie Hebammen für das ganze Gebiet des heutigen Kantons Waadt ausbildete. Allerdings beschränkte sich hier der Unterricht hauptsächlich auf Phantomkurse, da ein Spital nicht zur Verfügung stand. Einzig der Hebammenschule von Bern, der letzten der vier schweizerischen Hebammenschulen im 18. Jahrhundert, kam eine größere Bedeutung zu. Hier wurden für das große Kantonsgebiet (inklusive Aargau, der damals der bernischen Obrigkeit unterstand) in drei Jahren 54 Hebammen ausgebildet. Dem Hebammenmeister stand für die Ausbildung ein großes Spital zur Verfügung, so daß man wohl mit Recht behaupten darf, daß die bernische Hebammenschule im 18. Jahrhundert zwar die zuletzt gegründete, aber die bedeutendste war. Die Geschichte der Berner Hebammenschule ist aber noch aus einem anderen Grunde bemerkenswert. Es ist sicher nicht alltäglich, wenn drei so berühmte Ärzte wie Haller, Tissot und Herrenschwand aufgefordert wurden, zur Notwendigkeit und Organisation einer Hebammenschule ein Gutachten auszustellen. Damit bildet die vorliegende Arbeit zugleich einen biographischen Beitrag für diese drei Gelehrten, die das Ansehen der Schweiz im 18. Jahrhundert mitbegründet haben. 